INCIDENCIA DE LA POLÍTICA FISCAL VENEZOLANA SOBRE EL FENÓMENO INFLACIONARIO DURANTE 1997-2013 ${ }^{1}$

\title{
IMPACT OF FISCAL POLICY IN VENEZUELA ON THE PHENOMENON OF INFLATION DURING 1997-2013
}

\author{
Armando Urdaneta ${ }^{2}$ \\ Universidad Nacional Experimental Rafael María Baralt. (UNERMB)-Venezuela
}

RECIBIDO: Agosto 29 de 2014

ACEPTADO: Noviembre 02 de 2014

\section{Resumen}

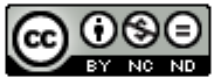

La presente investigación tiene como objetivo analizar la incidencia de la política fiscal venezolana sobre el fenómeno inflacionario durante el periodo 1997-2013, y surge de la necesidad de evaluar los instrumentos de política económica considerando ante un posible escenario de dolarización de la economía y el impacto que las variables de política fiscal pudiesen tener en el comportamiento del nivel de precios, según los planteamientos de Mochón (2008) y Guerra (2013) entre otros. La metodología es de tipo analítica y retrospectiva; en el diseño bibliográfico se utilizaron datos secundarios, tales como los informes económicos del Banco Central de Venezuela (BCV), atendiendo con especial detalle series de tiempo y suavización exponencial de las variables estudiadas en periodos trimestrales. De esta manera se concluye una baja y mediana correlación entre las variables sujetas a estudio, que da como resultado una correlación de 0,25 entre la tasa de inflación y el gasto de consumo final del Gobierno y de 0,61 entre los impuestos netos sobre sus propios productos del Gobierno y su gasto de consumo final; se termina obteniendo una nula correlación de 0,002 entre los impuestos netos sobre los productos del Gobierno y la tasa de inflación.

Palabras clave: Política fiscal, gasto público, política económica, tasa de inflación e impuesto sobre los productos.

\begin{abstract}
This research aims to analyze the impact of Venezuela's fiscal policy on the inflation phenomena during the period 1997-2013; the study arises from the need to evaluate policy instruments to be considered before a possible scenario of dollarization of the economy, and the impact that fiscal policy variables could have on the behavior of the price level. Such approach is supported in Mochon (2008) and War (2013) among others. The type of methodology is analytical and retrospective; on the bibliographic design side secondary data was used, such as the economic reports of the Central Bank of Venezuela (BCV), making a detailed study using time series and exponential smoothing of the variables under study data were used in Quarterly periods. A low and medium correlation between the variables subject to study was concluded, resulting in a correlation of 0.25 between the inflation rate and the final consumption expenditure of government and 0.61 between net taxes on products and government final consumption expenditure of government; to finally obtain a zero correlation of 0.002 between the net taxes on products of the government and the inflation rate.
\end{abstract}

Keywords: Fiscal policy, public expenditure, economic policy, inflation and taxes on products.

\section{Este artículo se puede referenciar}

Urdaneta, A. (2015). Incidencia de la política fiscal venezolana sobre el fenómeno inflacionario durante 1997-2013, en Desarrollo Gerencial Revista de la Facultad de Ciencias Económicas, administrativas y contables de la Universidad Simón Bolívar 7(1), 30-47.

\footnotetext{
${ }^{1}$ Proyecto de investigación: Lineamientos de política económica para la dolarización: una aproximación para la experiencia Venezolana. Universidad del Zulia-Venezuela. 2015.

${ }^{2}$ Ingeniero en Computación. Magister en Gerencia Empresarial, Magister en Telemática. Doctor en Ciencias Gerenciales. Candidato a doctor en Economía. Profesor universitario asociado a dedicación exclusiva del Programa de Administración de la Universidad Nacional Experimental Rafael María Baralt, Zulia-Venezuela. Email: ajum69@gmail.com
}

Desarrollo Gerencial, 7 (1) pp. 30 - 47 Enero-Junio 2015. ISSN: 2145-5147 (On Line). Universidad Simón Bolívar. Barranquilla-Colombia. Contactos: desarrollogerencial@unisimonbolivar.edu.co 


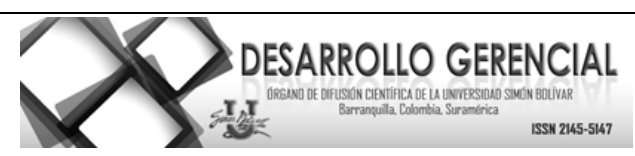

\section{1.- INTRODUCCIÓN}

El objetivo del presente estudio consiste en analizar la incidencia de la política fiscal venezolana en el fenómeno inflacionario durante el periodo 1997-2013; realizando una evaluación trimestral de las variables gasto del consumo final del Gobierno como componente de la demanda agregada interna; el impuesto neto sobre los productos como parte del producto interno bruto y la tasa de inflación: seguidamente se aplicó el método de series de tiempo en aras de determinar si las mismas presentan un componente tendencial, cíclico, estacional e irregular; luego se empleó el método de suavización exponencial para eliminar las fluctuaciones aleatorias en la serie de tiempo, con el objeto de proporcionar datos menos distorsionados al comportamiento regular de las variables, para al final realizar un análisis de correlación en la misma y evaluar el posible grado de asociación que existen entre las variables.

Ahora bien, la razón a la que obedece el presente estudio se debe a la importancia que tiene la política fiscal como instrumento de gestión pública por parte de los gobiernos para conducir y orientar la actividad económica de cada país y lograr a través suyo el cumplimiento de objetivos propuestos por el Estado, tales como generar un crecimiento económico y disminuir la tasa de desempleo sin que ello implique un impacto en la estabilidad de precios, conforme lo plantea Guerra (2013), pues se traduce en un incremento positivo del producto interno bruto per cápita y una distribución equitativa de la renta nacional entre otros. De este modo, las políticas fiscales implementadas por los gobiernos, en conjunto con otras políticas macroeconómicas, deben estar dirigidas a impactar favorablemente el bienestar social de la población, referido al mejoramiento progresivo de la calidad de vida de la sociedad.

En este sentido, el caso de la economía venezolana durante el periodo sujeto a estudio, muestra una política fiscal expansiva con déficit fiscal estructural, donde, por ejemplo, en términos reales, el gasto de consumo final del Gobierno ha aumentado 196,87\% mientras los impuestos netos sobre los productos han crecido 111,21\%, según la información estadística suministrada por el Banco Central de Venezuela (BCV) (2014). A ello se aúna el constante déficit fiscal, que ha variado entre un 5\% y $10 \%$ con respecto al producto interno bruto real (PIBR).

Este escenario de agudo déficit fiscal se ha visto acompañado por un aumento sostenido del nivel de precios como reflejo de una inflación acumulada de 3324,94\% en el periodo 1997-2013, mientras que el PIBR solo ha crecido un 48,75\%; es evidente entonces el comportamiento de una economía con altas tasas de inflación y bajos niveles de crecimiento, lo cual sin duda repercute en el poder compra real del bolívar y, por ende, en el nulo aumento de los salarios reales, o peor aún, en su paulatina disminución.

Por ello, es fundamental para los intereses de la presente investigación evaluar el comportamiento trimestral de algunas variables de política fiscal y su impacto en la tasa de inflación tal como lo plantean 


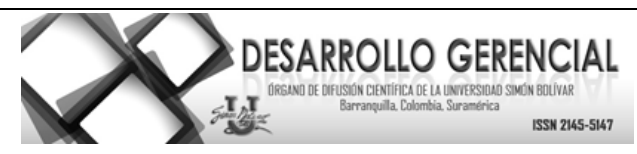

Cartaya, Sáez y Bolívar (2011), a fin de constatar si estas han contribuido a la construcción del bienestar social de la población, pues no se ha evidenciado un crecimiento económico sostenido, acompañado de una distribución de renta que favoreciendo el incremento del empleo, impida el aumento de los niveles de pobreza.

Para este estudio, la información analizada de las variables gasto de consumo final del Gobierno, impuestos netos sobre los productos y la tasa de inflación es trimestral. Por lo tanto, se realizó un análisis mediante las series de tiempo que Anderson, Sweeney y Williams (2008) definen como un conjunto de observaciones de una variable medida en puntos o periodos sucesivos en el tiempo, cuyo objetivo es proporcionar buenos pronósticos, o predicciones, de futuros valores de la serie de tiempo entre el gasto de consumo final del Gobierno, impuestos netos sobre los productos y la tasa de inflación trimestral.

En consecuencia, al realizar un análisis de la serie de tiempo de las variables gasto de consumo final del Gobierno, impuestos netos sobre los productos y la tasa de inflación trimestral, esta investigación busca determinar: ¿Existe una correlación de la política fiscal venezolana en el periodo 1997-2013 con el fenómeno inflacionario?, en aras de establecer una causalidad posible.

\section{Enfoque epistemológico}

En la presente investigación se establece un contraste teórico entre las posturas keynesianas y poskeynesianas desarrolladas por John Maynard Keynes durante la década de los años treinta luego de la Gran Depresión de la economía norteamericana, y quien según Ekelund y Hebert (2008) en su obra La teoría general hacía énfasis en el uso de la política fiscal por parte de los gobiernos para complementar los mecanismos de mercado del sector privado, en aras de resolver los problemas de desempleo existentes.

Keynes creía que probablemente el sector privado no haría las inversiones necesarias para aumentar la tasa de empleo, razón por la cual, según Ekelund y Hebert (2008), sugería que los gastos e impuestos del Gobierno actuaran como elementos compensadores a fin de aliviar el desempleo y la subproducción, pero se oponía en forma enérgica al uso del recurso exclusivo de la política monetaria para estabilizar la economía.

Por ello, Keynes (citado en Herrerías, 2011), pensaba que la inversión agregada privada estaba condicionada en gran manera por las expectativas sobre precios y beneficios, donde una disminución rápida del nivel de precios puede, prácticamente, reducir el nivel de inversión, incluso en el caso de los tipos de interés decrecientes. Las quiebras y condiciones adversas de los negocios seguirían casi con toda certeza a la disminución de los precios. 


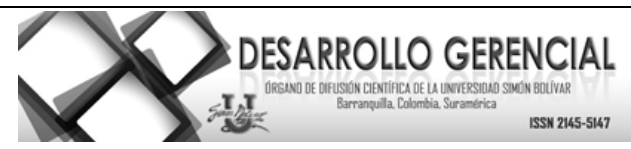

En este sentido, Keynes (citado en Ekelund \& Hebert, (2008) argumentaba sobre la base de sus desarrollos teóricos que el Gobierno debía usar sus poderes para gravar impuestos y para gastar, a fin de influir en el ciclo económico. El gasto gubernamental debía representar una inyección directa de inversión pública en el flujo de la renta, que podía financiarse mediante impuestos progresivos, reduciendo así el consumo, pero en cantidad menor a la que le corresponde el gravamen. Por ello proponía un programa planificado en gran escala de política fiscal discrecional, así como un fortalecimiento de los estabilizadores incorporados como impuestos progresivos. En pocas palabras, el Gobierno tenía que estar preparado para proporcionar las condiciones de pleno empleo.

En contraposición a los planteamiento anteriores, (Fernández, Rodríguez \& Parejo, 2008), se encuentra la corriente monetarista encabezada por el economista Milton Friedman, premio nobel de economía 1976, quien (citado en Fernández et al., 2008) plantea que la manipulación de las variables nominales no tiene un efecto sostenible sobre las variables reales a mediano y largo plazo, partiendo de la premisa del valor neutral del dinero desarrollado por David Hume en 1776 según Ekelund y Hebert (2008).

Por esta razón un aumento del gasto público orientado a estimular el crecimiento económico en los ciclos recesivos, como lo planteaba Keynes, solo traería consigo un incremento general de precios en el mediano y largo plazo, y si bien en corto plazo según señalaba Friedman se producía un leve crecimiento económico y una disminución del desempleo, en el mediano y largo plazo estos retornarían a la posición inicial donde comenzó la utilización de políticas fiscales y monetarias expansivas.

Para Friedman (citado en Ekelund \& Hebert, 2008), la economía es básicamente constante y autorregulada si el Gobierno y el banco de reserva federal tiene como papel proporcionar un entorno predecible y estable en el que los procesos económicos pueden funcionar sin trabas, con mayor eficiencia a fin de maximizar el bienestar económico. Así pues, la mínima intervención del Gobierno, los presupuestos equilibrados, desregulación de los precios y una norma de crecimiento monetario forman parte de las premisas de los monetaristas.

Otra teoría contemporánea desarrollada por los monetaristas es la llamada hipótesis de las expectativas racionales, la cual plantea que los participantes en el mercado no ignoran ni desechan información acerca de las predicciones sobre el curso futuro de la actividad económica, en aras de anticipar racionalmente los efectos de la políticas fiscales del Gobierno, reaccionando en el presente con las expectativas que se han formado. En caso de que los consumidores de bienes y servicios e instrumentos financieros y los productores reaccionaran a las políticas económicas discrecionales, mediante el aprendizaje de los efectos y acciones en ellas implícito conforme sus expectativas racionales, se neutralizaría total o parcialmente los efectos deseados de las medidas. 
En el caso venezolano al igual que en la mayoría de los países de América Latina según Manzano et al. (2008), los gobiernos se han caracterizado por utilizar medidas de expansión fiscal y monetaria en aras de estimular el crecimiento económico, pero con frecuencia fracasan en su propósito de mantener la inflación dentro de los límites de moderación y control.

Esto se debe a que la expansión del gasto público supera los ingresos fiscales, y genera constantes y agudos déficits fiscales, los cuales son financiados mediante el endeudamiento interno y externo, pero en la mayoría de los casos mediante expansión de la masa monetaria, la cual al crecer muy por encima de la renta real de la economía produce un desequilibrio entre el mercado de bienes y el mercado de dinero, y siendo los precios según Manzano, et al. (2008) una relación proporcional entre la cantidad de bienes y servicios que produce la economía y la cantidad de dinero circulante, al incrementarse esta última se produce un aumento general de precios de los productos y servicios.

Es por ello que Hayek (1976), (citado en Ekelund y Hebert, 2008), plantea que la inflación distorsiona la normal asignación de ahorro e inversión hacia necesidades más acuciantes, pues provoca ineficiencia en su asignación. Las tensiones inflacionistas producidas sobre los bienes de consumo presente, se traducirán en una elevación del tipo de interés del mercado que traerá serias dificultades a las empresas proveedoras, dada la elevación de sus costos de producción, en especial los laborales. Mientras los empresarios atentos a generar beneficios, fracasan en sus apreciaciones cuando estudian un proyecto de inversión, puesto que los precios relativos de sus factores de producción están alterados sobre todo la tasa interna de retorno de la inversión, la cual mide si un proyecto es rentable o no.

\section{Conceptualización de la Política Fiscal}

De acuerdo con lo planteado por el ABC Económico del BCV (2012), las políticas fiscales constituyen instrumentos de gestión pública que utilizan los gobiernos para conducir y orientar la actividad económica del país y lograr a través suyo el cumplimiento de objetivos que se propone el Estado, como son: generar desarrollo económico mediante el crecimiento positivo del producto interno bruto (PIB), el incremento del producto interno bruto per cápita y una distribución equitativa de la renta nacional entre otros. De este modo, las políticas fiscales implementadas por los gobiernos lo mismo que otras políticas macroeconómicas deben actuar conjuntamente para impactar el bienestar social de la población, en términos de mejoramiento progresivo de la calidad de vida de la sociedad.

Según Fernández, Rodríguez y Parejo (2008), la política fiscal es el conjunto de variaciones en los programas de gastos y de ingresos del Gobierno, realizados con el fin de colaborar al logro de los objetivos de la política macroeconómica. Mediante estas variaciones se piensa que el o los gobiernos 


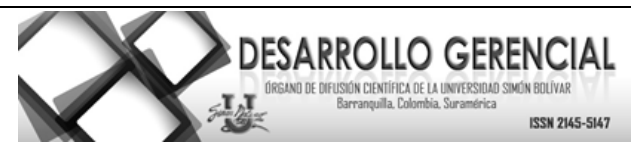

ejercen un poderoso impacto sobre la demanda agregada y, por consiguiente, dado un nivel de precios, sobre la producción y el empleo. En definitiva, la política fiscal constituye la vía más importante para mantener o mejorar el llamado Estado del bienestar (que se caracteriza políticamente, como una actuación solidaria de la sociedad que trata de garantizar cierto nivel de vida a los ciudadanos, poniendo una red protectora que aminore desigualdades).

Al respecto Toro (1993) plantea que la política fiscal es el instrumento mediante el cual el Estado realiza sus funciones de gasto público, impuestos, estabilización y planificación de la política económica de la nación. La política fiscal aplicada en Venezuela ha sido mal manejada a través de los años; en consecuencia, hoy en día el país presenta una de los déficits fiscales más altos de Latinoamérica y se encuentra en un proceso de estancamiento e inflación del cual resulta difícil, más no imposible salir.

En este mismo orden de ideas, Zabala y González (1992), describen que el propósito de la política fiscal es darle mayor estabilidad al sistema económico, mientras consigue el objetivo de ocupación plena, manteniendo o buscando un punto de equilibrio entre gasto fiscal y el ingreso fiscal, en aras de mantener el balance macroeconómico sin grandes inclinaciones en el empleo ni en la actividad económica, y previniendo severos ataques de inflación a la vez que asegura una adecuada tasa de crecimiento económico mediante una apropiada distribución de la renta entre los diversos grupos de la economía, y una eficiente asignación de recursos en toda la economía.

En el caso venezolano, como se ha señalado anteriormente, la política fiscal ha sido de carácter expansivo, y presenta un déficit fiscal estructural que ha crecido $307 \%$ en términos reales a lo largo del periodo sujeto a estudio, pasando del 5\% al 10\% del PIBR. Lo anterior debido a que los ingresos fiscales por concepto de impuestos netos sobre los productos y los derechos de importación como componentes del PIBR en la oferta global de la economía solo han crecido $140,18 \%$, en términos reales, mientras el gasto al consumo final del Gobierno como parte de la demanda agregada interna ha crecido 196,87\%. Según el BCV (2014), esto ha producido un aumento de la masa monetaria para financiar las dos terceras parte del déficit público, y el restante ha sido por la vía del endeudamiento interno y externo de la nación.

Este incremento de la masa monetaria, sin la debida correspondencia con el aumento de la renta real se ha traducido en un aumento general de los precios y con ello de la inflación, la cual presenta crecimiento promedio anual del 33,76\% según el BCV (2014); a su vez, el PIBR solo muestra un avance promedio anual del $3,02 \%$, mientras que la tasa desempleo ha presentado un comportamiento promedio del $10 \%$. 


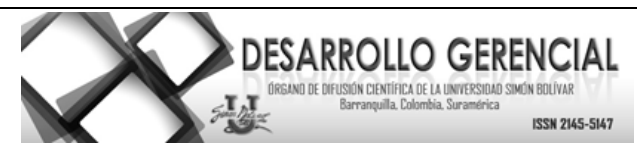

\section{MÉTODO}

El presente estudio considera que el paradigma que mejor lo caracteriza es el positivismo, el cual, según Martínez (2013), se fundamenta en el auge de la lógica apoyada en las matemáticas que buscaban convertir esta disciplina filosófica en el método adecuado para el conocimiento de la realidad y la nueva metodología de verificación científica. Wittgenstein (1926), tomado de Blaxter, Hugues y Tight (2009), parte de los principios del simbolismo y de las relaciones entre las palabras y las cosas en cualquier lenguaje, y aplica el resultado de esta investigación a las diferentes ramas de la filosofía tradicional como idea central de su tesis sosteniendo que una proposición es una imagen o figura, pintura de la realidad.

La presente investigación es de carácter documental lo cual según Bernal (2006) consiste en un análisis de la información escrita, con el propósito de establecer relaciones, diferencias, etapas, posturas o estado actual del conocimiento respecto al comportamiento de la política fiscal venezolana en el periodo sujeto a estudio; al mismo tiempo el objetivo del presentado es propio de una investigación correlacional, que según Díaz (2009) busca evaluar el grado de relación entre dos variables: en este caso de la política fiscal, medida a través del gasto al consumo final del Gobierno y los impuestos netos sobre los productos, con la tasa de inflación.

Por otra parte, atendiendo a Nieto y Rodríguez (2009) se siguió un diseño no experimental debido a que no hubo manipulación de las variables objeto de investigación, se procedió a su igualación, aplicando métodos estadísticos de serie de tiempo, suavización exponencial y correlación lineal de Pearson para estudiar estadísticamente el comportamiento y relación de las variables.

A continuación se puede afirmar que dicha investigación se enmarca dentro del paradigma positivista, basado, según Blaxter, Hugues y Tight (2009), en los planteamientos de Auguste Comte; se caracteriza por validar solo aquellos conocimientos provenientes de las ciencias empíricas, y hacer de esta disciplina filosófica el método adecuado para el conocimiento de la realidad y una nueva forma de verificación científica. De ahí según Blaxter, Hugues y Tight (2009), emergieron filósofos de la talla de Wittgenstein (1926), Russell y Whitehead (1936), con importantísimas aportaciones al desarrollo de esta nueva lógica. El objetivo era convertir las proposiciones del lenguaje en módulos a través de los cuales puede encerrarse la realidad.

La verificación de estas debía hacer posible saber qué proposiciones pueden ser dichas y tener sentido y cuáles no. Por lo cual se investiga la realidad que se evidencia en las políticas fiscales implementadas en la economía venezolana en el periodo 1997-2013, con el propósito de constatar según los agregados macroeconómicos publicados por el $\mathrm{BCV}$, el impacto o la incidencia que esta tiene en términos de las variables reales de la economía en el fenómeno inflacionario que la misma economía padece. 
Análisis de la serie de tiempo trimestral para la variable del gasto de consumo final del Gobierno

El gasto de consumo final de Gobierno según BCV (2014) son las compras de bienes, servicios y transferencias realizadas por los organismos públicos o el Estado. Estos, precisa Mochón (2008), incluye en términos reales el volumen de todos los gastos corrientes para la adquisición de bienes y servicios, remuneración de los empleados, gastos en defensa y formación de capital del Gobierno.

\section{Gráfico 1: Gasto de consumo final del gobierno a precios constantes de 1997 (miles de bolívares)}

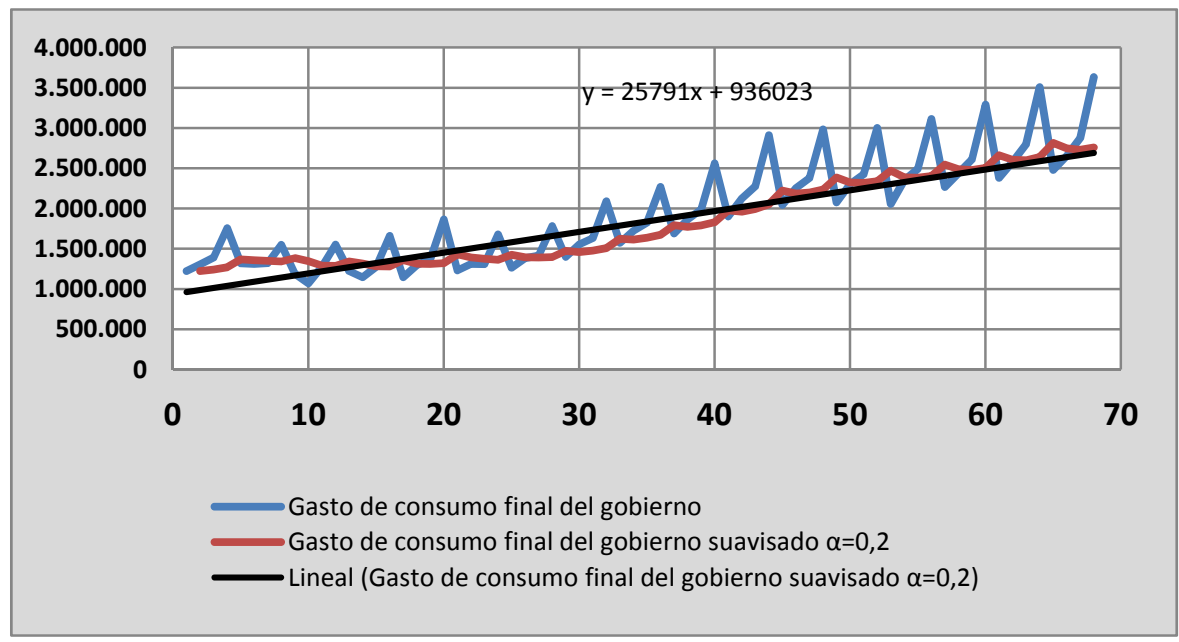

Fuente: Banco Central de Venezuela (2014).

Anderson, Sweeney y Williams (2008) muestran que la variable gasto del consumo final del Gobierno, fijada en el gráfico 1, presenta una tendencia lineal creciente en los 68 trimestres estudiados; al mismo tiempo se evidencia un comportamiento cíclico en donde los valores trimestrales oscilan por encima y por debajo de la línea de tendencia en una forma recurrente. Esta línea de tendencia no es más que la función de regresión de la variable antes señalada en función de tiempo por ser fundamental característica de las series de tiempo en donde la variable dependiente es el tiempo y la independiente en este caso es la variable estudiada.

En este mismo orden de ideas la serie de tiempo antes señalada muestra un componente estacional, que en promedio decrece en un $27 \%$ en el primer trimestre del año aun cuando mantiene constante su evolución con un aumento de 7\% durante los 2 siguientes trimestres, para luego incrementarse en el cuarto trimestre en un $26 \%$, todo ello en valores promedios de la serie, a los efectos de la variable estudiada: la función de regresión. Se representa la línea de tendencia siguiente:

\section{Ecuación de una tendencia lineal}

$$
T_{t=} b_{0}+b_{1} t
$$


Donde:

$\boldsymbol{T}_{\boldsymbol{t}}=$ Valor de la tendencia de la serie de tiempo en el periodo $\mathrm{t}$

$\boldsymbol{b}_{\mathbf{0}}=$ ordenada al origen de la línea de tendencia

$\boldsymbol{b}_{\mathbf{1}}=$ pendiente de la línea de tendencia

$\boldsymbol{t}=$ tiempo

Cálculo de la pendiente $\left(b_{1}\right)$ y la ordenada al origen $b_{0}$

$$
\begin{gathered}
b_{1}=\frac{\sum t Y_{t}-\left(\sum t \sum Y_{t}\right) / n}{\sum t^{2}-\left(\sum t^{2}\right) / n}=25791 \\
b_{o=} \bar{Y}+b_{1} \bar{t}=936023
\end{gathered}
$$

$T_{t}=25791 t+936023$

Donde:

$\boldsymbol{Y}_{\boldsymbol{t}}=$ Valor de la serie de tiempo en el periodo $\mathrm{t}$

$\boldsymbol{n}=$ número de periodos

$\overline{\boldsymbol{Y}}=$ valor promedio de la serie de tiempo, $\bar{Y}=\sum Y_{t} / n$

$\overline{\boldsymbol{t}}=$ valor promedio de tiempo de $\overline{\boldsymbol{t}} ;=\sum Y_{t} / n$

La ecuación antes planteada se obtuvo mediante la estimación de regresión lineal simple, mediante la suavización exponencial de los promedios ponderados de la serie de tiempo, seleccionando un peso o factor de ponderación conocido como $\alpha$ o constante de suavización, el cual se representa $(0 \leq \alpha \leq 1)$. Dicho modelo de suavización se muestra a continuación:

$$
F 1=\propto Y t+(1 \propto) F 1
$$

Donde

$\mathrm{Ft}+1=$ pronóstico de la serie de tiempo para el periodo $\mathrm{t}+1$

$\mathrm{Yt}=$ valor real de la serie de tiempo en el periodo $\mathrm{t}$

$\mathrm{F} 1=$ pronóstico de la serie de tiempo para el periodo $\mathrm{t}$

$\alpha=$ constante de suavizamiento $(0 \leq \alpha \leq 1)$

En función del modelo de suavización antes expuesto, para las estimaciones hechas a las diferentes constantes de suavización, se determinó como la función de suavización ideal aquella en la cual el valor de $\alpha$ es 0.2 debido a que esta presenta la menor desviación estándar de error absoluto típico muestral; así, la curva suavizada permitió establecer un mecanismo de autocorrección para ajustar los pronósticos en dirección opuesta a las perturbaciones pasadas, y en función de ello calcular una función de regresión más óptima. 
Análisis de la serie de tiempo trimestral para la variable de los impuestos netos sobre los productos del Gobierno

Impuestos netos sobre los productos, según el BCV (2014), son ingresos impositivos que recauda el Gobierno menos las trasferencias y subsidios que reciben las familias y empresas. Estos, indica Mochón (2008), son la suma de impuestos a los productos menos los subsidios, donde los impuestos a los productos son los gravámenes pagaderos por parte de productores relacionados con las áreas de producción, venta, compras o uso de bienes y servicios. Los subsidios son donaciones a la cuenta corriente otorgadas por el Gobierno a empresas privadas y públicas no constituidas en sociedad. Las donaciones pueden adquirir la forma de pagos para asegurar un precio mínimo o para permitir que se mantengan los precios de bienes y servicios a niveles inferiores a los costos de producción, y otras formas de ayuda a los productores.

Gráfico 2: Impuestos netos sobre los productos del gobierno a precios constantes de 1997 (miles de bolívares).

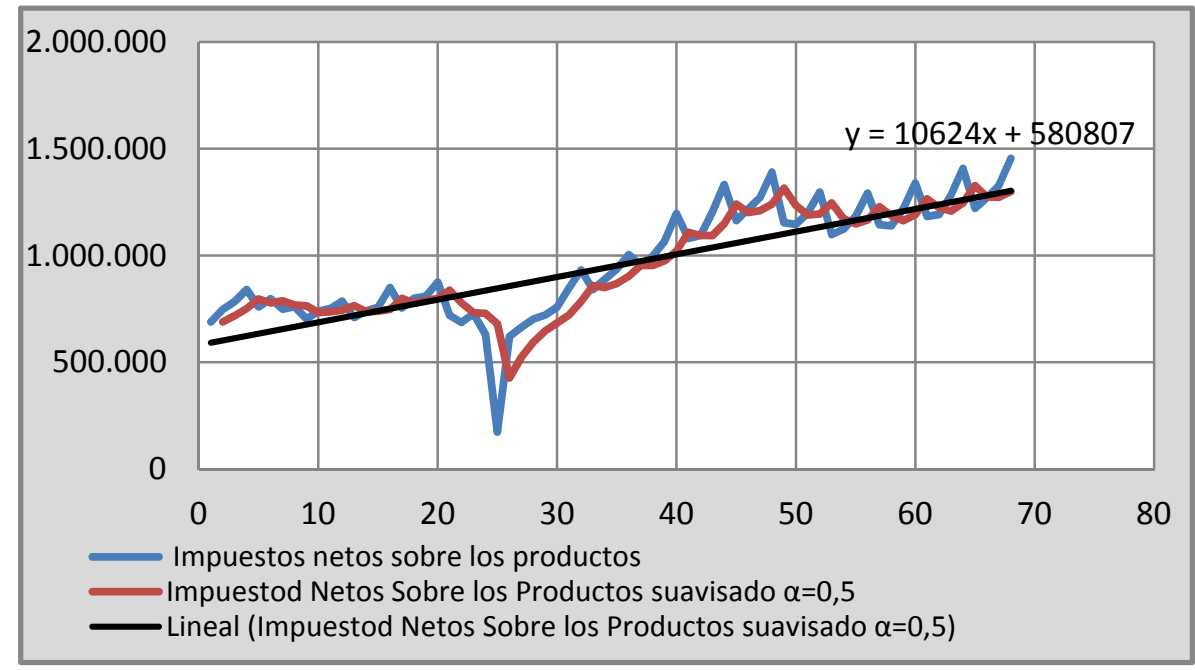

Fuente: Banco Central de Venezuela (2014).

Según Anderson, Sweeney y Williams (2008), en el gráfico 2 se expresa que los impuestos netos sobre los productos presentan una tendencia lineal creciente en los 68 trimestres estudiados; al mismo tiempo se evidencia un comportamiento cíclico en los cuales los valores trimestrales oscilan por encima y por debajo de la línea de tendencia en una forma recurrente. Dicha línea de tendencia no es más que la función de regresión de la variable antes señalada en función de tiempo por ser esta una característica fundamental de las series de tiempo en el cual la variable dependiente es el tiempo y la independiente en este caso es la variable estudiada.

En este mismo orden de ideas, la serie de tiempo antes señalada muestra un componente estacional, el cual decrece en promedio un $15 \%$ en el primer trimestre del año y aumenta en promedio un $18 \%$ en el 


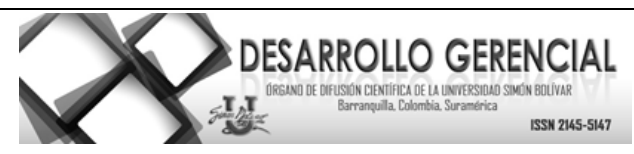

segundo trimestre, para posteriormente oscilar entre un el 5\% y 7\% en los 2 trimestres restantes de cada año; para los efectos de la variable estudiada, la función de regresión que representa la línea de tendencia es la siguiente:

\section{Ecuación de una tendencia lineal}

$$
T_{t=} b_{0}+b_{1} t
$$

Donde

$\boldsymbol{T}_{\boldsymbol{t}}=$ Valor de la tendencia de la serie de tiempo en el periodo $\mathrm{t}$

$\boldsymbol{b}_{\mathbf{0}}=$ ordenada al origen de la línea de tendencia

$\boldsymbol{b}_{\mathbf{1}}=$ pendiente de la línea de tendencia

$\boldsymbol{t}=$ tiempo

\section{Cálculo de la pendiente $\left(b_{1}\right)$ y la ordenada al origen $b_{0}$}

$$
\begin{gathered}
b_{1}=\frac{\sum t Y_{t}-\left(\sum t \sum Y_{t}\right) / n}{\sum t^{2}-\left(\sum t^{2}\right) / n}=10624 \\
b_{o=} \bar{Y}+b_{1} \bar{t}=580807
\end{gathered}
$$

$T_{t}=10624 t+580807$

Donde

$\boldsymbol{Y}_{\boldsymbol{t}}=$ Valor de la serie de tiempo en el periodo $\mathrm{t}$

$\boldsymbol{n}=$ número de periodos

$\overline{\boldsymbol{Y}}=$ valor promedio de la serie de tiempo, $\bar{Y}=\sum Y_{t} / n$

$\overline{\boldsymbol{t}}=$ valor promedio de tiempo de $\overline{\boldsymbol{t}} ;=\sum Y_{t} / n$

La ecuación antes planteada se obtuvo mediante la estimación de regresión lineal simple, utilizando la suavización exponencial de los promedios ponderados de la serie de tiempo, seleccionando un peso o factor de ponderación conocido como $\alpha$ o constante de suavización, el cual se representa $(0 \leq \alpha \leq 1)$; dicho modelo de suavización se muestra a continuación:

$$
F 1=\propto Y t+(1 \propto) F 1
$$

Donde

$\mathrm{Ft}+1=$ pronóstico de la serie de tiempo para el periodo $\mathrm{t}+1$

$\mathrm{Y} \mathrm{t}=$ valor real de la serie de tiempo en el periodo $\mathrm{t}$

$\mathrm{F} 1=$ pronóstico de la serie de tiempo para el periodo $\mathrm{t}$

$\alpha=$ constante de suavizamiento $(0 \leq \alpha \leq 1)$ 


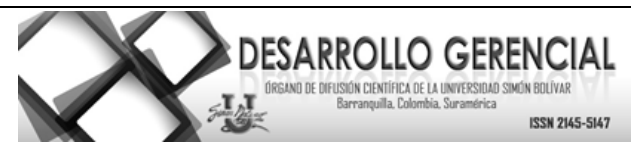

En función del modelo de suavización antes expuesto, las estimaciones hechas para las diferentes constantes de suavización determinó como la función de suavización ideal aquella en la cual el valor de $\alpha$ es 0.5 debido a que esta presenta la menor desviación estándar de error absoluto típico muestral, donde la curva suavizada permitió establecer un mecanismo de autocorrección para ajustar los pronósticos en dirección opuesta a las perturbaciones pasadas, y en función de ello calcular una función de regresión más óptima.

Análisis de la serie de tiempo trimestral para la tasa de inflación de la economía venezolana en el periodo 1997-2013

Según el BCV (2014), tasa de inflación es la variación porcentual que se obtiene al comparar entre periodos los resultados del índice utilizado para medir la inflación. En Venezuela, al igual que en muchos países, suele utilizarse el índice de precios al consumidor para medir la tasa de inflación. En este sentido Mochón (2008) afirma que la misma es una medida de la tasa de crecimiento anual del deflactor implícito del producto interno bruto (PIB), el cual muestra la tasa de variación de precios en la economía en general; donde el deflactor del PIB es el cociente entre el PIB en moneda local a precios corrientes y el PIB en moneda local a precios constantes.

En este mismo sentido, Fernández, Rodríguez y Parejo (2008) expresan que la inflación es causada también por un incremento de los componentes de los costos de producción, tales como salarios, impuestos, beneficios, intereses y devaluación de la moneda, provocados por perturbaciones en la oferta agregada, aunado a una expansión del nivel del gasto interno de la economía, principalmente del consumo de familias y del gasto del Gobierno, los cuales conjuntamente con los niveles de inversión directa producen cambios significativos en la demanda agregada interna.

Gráfico 3: Tasa de inflación trimestral de la economía venezolana en periodo 1997-2013

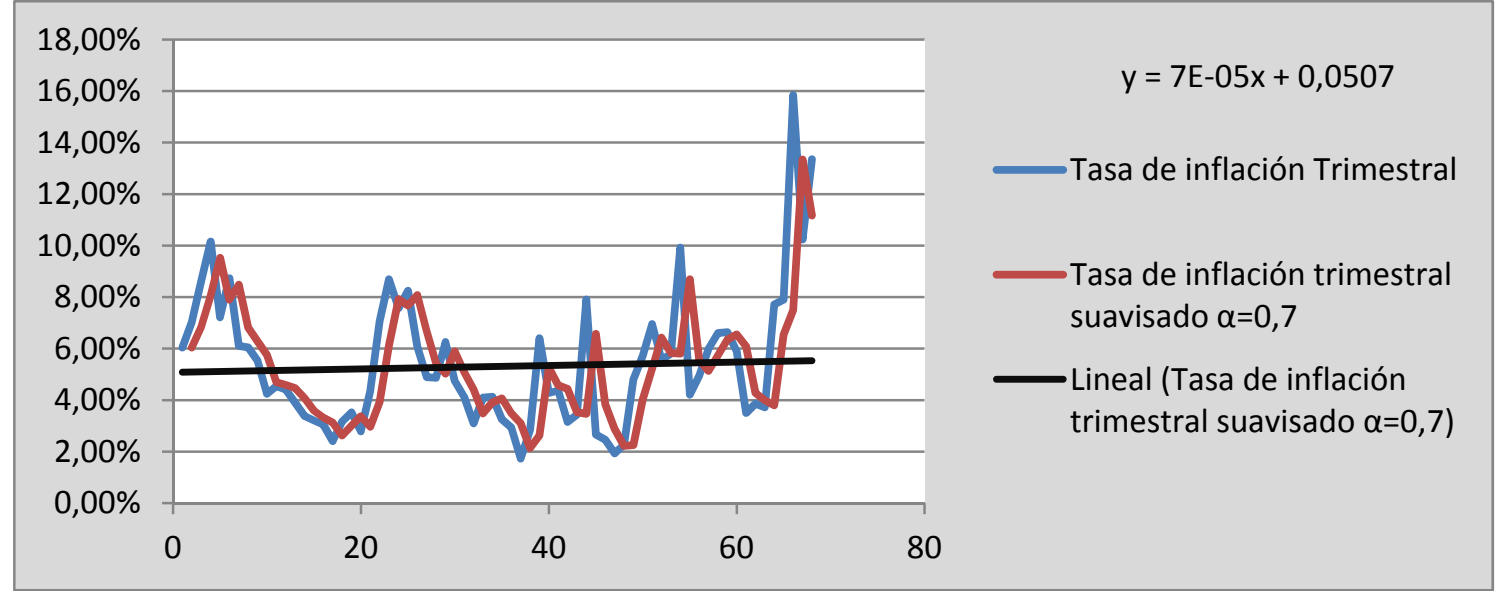

Fuente: Banco Central de Venezuela (2014). 


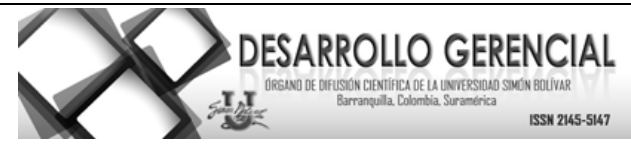

Según Anderson, Sweeney y Williams (2008), en el periodo 1997 -2013, la tasa de inflación trimestral de la economía venezolana no presenta una tendencia lineal creciente o decreciente en los 68 trimestres observados en el gráfico 3; al mismo tiempo se evidencia un comportamiento cíclico en el que los valores trimestrales oscilan por encima y por debajo de la línea de tendencia en una forma recurrente. Esta línea de tendencia no es más que la función de regresión de la variable antes señalada en función de tiempo, por ser esta una característica fundamental de las series de tiempo en la cual la variable dependiente es el tiempo y la independiente, en este caso, es la variable estudiada.

En este mismo orden de ideas la serie de tiempo antes señalada no muestra un componente estacional, sino que la misma oscila entre un 5\% y un $6 \%$ durante los cuatro trimestres de cada año; para los efectos de la variable estudiada, la función de regresión que representa la línea de tendencia es la siguiente:

\section{Ecuación de una tendencia lineal}

$$
T_{t=} b_{0}+b_{1} t
$$

Donde

$\boldsymbol{T}_{\boldsymbol{t}}=$ Valor de la tendencia de la serie de tiempo en el periodo $\mathrm{t}$

$\boldsymbol{b}_{\mathbf{0}}=$ ordenada al origen de la línea de tendencia

$\boldsymbol{b}_{\mathbf{1}}=$ pendiente de la línea de tendencia

$\boldsymbol{t}=$ tiempo

\section{Cálculo de la pendiente $\left(b_{1}\right)$ y la ordenada al origen $b_{0}$}

$$
\begin{gathered}
b_{1}=\frac{\sum t Y_{t}-\left(\sum t \sum Y_{t}\right) / n}{\sum t^{2}-\left(\sum t^{2}\right) / n}=7 E-05 \\
b_{o=} \bar{Y}+b_{1} \bar{t}=0,0507
\end{gathered}
$$

Donde

$\boldsymbol{Y}_{\boldsymbol{t}}=$ Valor de la serie de tiempo en el periodo $\mathrm{t}$

$\boldsymbol{n}=$ número de periodos

$\overline{\boldsymbol{Y}}=$ valor promedio de la serie de tiempo, $\bar{Y}=\sum Y_{t} / n$

$\overline{\boldsymbol{t}}=$ valor promedio de tiempo de $\overline{\boldsymbol{t}} ;=\sum Y_{t} / n$

$\mathrm{T}_{\mathrm{t}}=7 \mathrm{E}-05 \mathrm{t}+0,0507$

La ecuación antes planteada se obtuvo mediante la estimación de regresión lineal simple, utilizando la suavización exponencial de los promedios ponderados de la serie de tiempo, seleccionando un peso o factor de ponderación conocido como $\alpha$ o constante de suavización, el cual se representa $(0 \leq \alpha \leq 1)$; dicho modelo de suavización se muestra a continuación: 


$$
F 1=\propto Y t+(1 \propto) F 1
$$

Donde:

$\mathrm{Ft}+1=$ pronóstico de la serie de tiempo para el periodo $\mathrm{t}+1$

$\mathrm{Yt}=$ valor real de la serie de tiempo en el periodo $\mathrm{t}$

$\mathrm{F} 1=$ pronóstico de la serie de tiempo para el periodo $\mathrm{t}$

$\alpha=$ constante de suavizamiento $(0 \leq \alpha \leq 1)$

En función del modelo de suavización antes expuesto, las estimaciones hechas para las diferentes constantes de suavización se determinó como la función de suavización ideal es aquella en la cual el valor de $\alpha$ es 0.7 debido a que esta presenta la menor desviación estándar de error absoluto típico muestral, donde la curva suavizada permitió establecer un mecanismo de autocorrección para ajustar los pronósticos en dirección opuesta a las perturbaciones pasadas, y en función de ello calcular una función de regresión más óptima.

\section{3.- RESULTADOS}

Para obtener los resultados, el estudio previamente evaluó el comportamiento de una serie de estadísticos descriptivos respecto a las variables objeto de investigación mediante la estimación de medidas de tendencia central, para posteriormente realizar el análisis de correlación en aras de establecer una relación causal entre las mismas. A continuación, en la tabla 1 se muestran los resultados.

Tabla 1. Estadísticos descriptivos

\begin{tabular}{|c|c|c|c|c|c|c|c|}
\hline & & $\begin{array}{c}\text { Valor } \\
\text { mínimo }\end{array}$ & $\begin{array}{c}\text { Valor } \\
\text { máximo }\end{array}$ & \multicolumn{2}{|c|}{ Media } & $\begin{array}{c}\text { Desviación } \\
\text { estándar }\end{array}$ & Varianza \\
\cline { 2 - 8 } & $\begin{array}{c}\text { Muestra } \\
\text { estadística }\end{array}$ & Estadístico & Estadístico & Estadístico & $\begin{array}{c}\text { Estánor } \\
\text { estar de } \\
\text { la media }\end{array}$ & Estadístico & Estadístico \\
\hline $\begin{array}{c}\text { Tasa de } \\
\text { inflación }\end{array}$ & 68 & $2,00 \%$ & $16,00 \%$ & $5,46 \%$ & $0,33 \%$ & $2,70 \%$ & $0,07 \%$ \\
\hline $\begin{array}{c}\text { Gasto de } \\
\text { consumo final } \\
\text { del Gobierno }\end{array}$ & 68 & $-34,00 \%$ & $36,00 \%$ & $3,64 \%$ & $2,40 \%$ & $19,66 \%$ & $3,87 \%$ \\
\hline $\begin{array}{c}\text { Impuestos } \\
\text { netos sobre los } \\
\text { productos del } \\
\text { Gobierno }\end{array}$ & 68 & $-73,00 \%$ & $259,00 \%$ & $4,24 \%$ & $4,14 \%$ & $33,87 \%$ & $11,47 \%$ \\
\hline
\end{tabular}

Fuente: Cálculos en SPSS V20 con base en los agregados macroeconómicos publicados por el BCV (2014). 


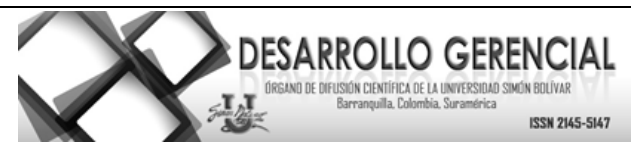

Los datos mostrados en la tabla 1, evidencian considerables disparidades entre las variables, pues la variación mínima trimestral en el periodo sujeto a estudio de la tasa de inflación es del $2 \%$, mientras que en el gasto de consumo final del Gobierno es 34\% y en los impuestos y netos sobre los productos $73 \%$. En cuanto a la variación máxima trimestral de la tasa de inflación: es del 16\%, mientras que en el gasto de consumo final del Gobierno es $36 \%$ y en los impuestos y netos sobre los productos $259 \%$.

En cuanto al comportamiento de la media de las variaciones trimestrales de las variables sujeta a estudio, en el periodo 1997-2013, estas presentan un comportamiento muy similar a la tasa de inflación, que es del 5,46\%, mientras que en el gasto de consumo final del Gobierno es 3,64\% y en los impuestos y netos sobre los productos 4,24\%. Sumado a ello el error estándar de la media, en las variaciones trimestrales muestran discrepancias significativas de $0,33 \%$ para la tasa de inflación; $2,40 \%$ para el gasto de consumo final del Gobierno y 4,14\% los impuestos y netos sobre los productos, con lo cual se demuestra variabilidades disímiles entre la estimación de la media verdadera y la estadística para cada una de las variables.

En cuanto a la desviación estándar, que mide la dispersión ascendente y descendente de los datos con respecto a la media se pudo constatar diferencias significativas entre las variables objeto de estudio en las variaciones trimestrales, siendo la estimación de $\pm 2,7 \%$ para la tasa de inflación, $\pm 19,66 \%$ para el gasto de consumo final del Gobierno y en los impuestos y netos sobre los productos $\pm 33,87 \%$. En este mismo orden de ideas, la evaluación de la varianza en las variaciones trimestrales de las variables sujetas a estudio, muestran considerables discrepancias de 0,07\%, mientras que en el gasto de consumo final del Gobierno es 3,87\% y en los impuestos y netos sobre los productos es $11,47 \%$;

En este sentido, después de realizar el análisis de correlación lineal de Pearson y luego aplicar la corrección de Sperman y Brown, da una baja y mediana correlación entre las variables sujetas a estudio, que produce como resultado una correlación de 0,18 entre la tasa de inflación y el gasto de consumo final del Gobierno y de 0,43 entre los impuestos netos sobre los productos del Gobierno y el gasto de consumo final del Gobierno; para finalmente obtener una nula correlación de 0,002 entre los impuestos netos sobre los productos del Gobierno y la tasa de inflación.

Ahora bien, al hacer el mismo análisis de correlación lineal de Pearson y luego aplicar la corrección de Sperman y Brown a las valor de las variables sujetas a estudio, pero suavizadas mediante el método de suavización exponencial para eliminar las perturbaciones, se consiguieron resultados muy similares a los expuestos anteriormente: una mediana correlación de 0,61 entre los impuestos netos sobre los productos del Gobierno y su gasto de consumo final; una baja correlación de 0,25 entre la tasa de inflación y el gasto de consumo final del Gobierno; así como una nula correlación de 0,005 entre los impuestos netos sobre los productos del Gobierno y la tasa de inflación.

Desarrollo Gerencial, 7 (1) pp. 30 - 47 Enero-Junio 2015. ISSN: 2145-5147 (On Line). Universidad Simón Bolívar. 


\section{4.- DISCUSIÓN}

Luego de lo descrito en el punto anterior se puede concluir firmemente que el comportamiento trimestral de las variables reales en el manejo de la política fiscal, tales como el gasto de consumo final del Gobierno y los impuestos netos sobre sus propios productos, no tiene un alto impacto en el fenómeno inflacionario en Venezuela, ya que este obedece a otros elementos de la política económica, como el incremento de la masa monetaria para financiar el estructural déficit fiscal, según plantea Guerra (2008), y que se puede evidenciar en términos reales en la medida en que pasó de 5\% del PIBR en 1997 a 10\% del PIBR en 2013, con un crecimiento en términos reales en el periodo sujeto a estudio de $307 \%$, producto de un aumento del 196,87\% del gasto de consumo final del Gobierno, mientras los impuestos netos sobre los productos del Gobierno solo subieron 111,21\% .

En este sentido también es importante resaltar, sobre lo expresado por Cartaya, Sáez y Bolívar (2011), la estructura del gasto público en Venezuela medido en términos nominales; así, de acuerdo a cifras oficiales de (Onapre) Oficina Nacional de Presupuesto (2014): el gasto corriente en promedio en el periodo 1997-2013 representó un 73\% del gasto público, mientras que solo el 21\% se dedicó al gasto del capital y el 6\% restante a las aplicaciones financieras. Sin duda ello estimula una mayor propensión marginal al consumo en lugar de la inversión, lo cual se traduce en una mayor demanda de bienes y servicios y, por ende, de inflación.

En conclusión, pudiera esgrimirse que todas las variables objeto de estudio a través de aplicación de series de tiempo presentan componentes cíclicos, mas no de tipo tendencial o estacional que para los efectos del estudio solo se constató en las variables gasto de consumo final del Gobierno y los impuestos netos sobre los productos de aquel, lo cual demuestra que a pesar del crecimiento en términos reales de dichas variables, estas no tienen incidencia directa en las variaciones trimestrales de la tasa de inflación.

En este sentido, también es importante destacar que cuando se realizan las estimaciones promedio de la serie de tiempo, cada una de las variables en cada uno de los trimestres manifiestan un comportamiento muy similar en el tercer trimestre del año, pero divergente en el resto de los periodos; en el caso del gasto de consumo final del Gobierno presentan una evolución promedio muy similar en el segundo y tercer trimestre; en el caso de los impuestos netos sobre los productos del Gobierno ocurre en el tercero y cuarto trimestre.

Sin embargo, cuando se analizan las medidas de dispersión de los datos como la varianza y la desviación estándar, se pueden establecer considerables discrepancias en las variaciones trimestrales de las variables sujeta a estudio, lo cual sin duda permite evidenciar la baja o nula correlación entre las variables de política fiscal y la tasa de inflación, y una mediana correlación entre ellas. 


\section{5.- REFERENCIAS}

Anderson, Sweeney \& Williams. (2008). Métodos cuantitativos para los negocios (9. ${ }^{a}$ ed.). México: Editorial Thompson.

Banco Central de Venezuela. (2013). Informe Estadístico. Extraído el 30 de abril de 2014 desde http://www.bcv.org.ve/c2/indicadores.asp.

Banco Central de Venezuela. (2012). ABC Económico. Extraído el 3 de julio de 2012 desde http://www.bcv.org.ve/c1/abceconomico.asp.

Banco Central de Venezuela. (2014). Sistema de Cuentas Nacionales. Extraído el 29 de marzo de 2014 desde http://www.bcv.org.ve/cuadros/series/ctasnac0810b97/ctasnac0810b97.asp?id=453.

Bernal, C. (2006). Metodología de la investigación: para Administración, Economía, Humanidades y Ciencias Sociales. México: Pearson Education.

Blaxter, L. Hugues, C. \& Tight, M (2009). Cómo se investiga. España: Editorial Graó.

Cartaya, V.; Sáez, F. \& Bolívar, W. (2011). Impacto de la política fiscal y monetaria en el ciclo económico de Venezuela. Un enfoque bayesiano. Ediciones Banco Central de Venezuela. CaracasVenezuela.

Díaz, V. P. (2009). Metodología de la Investigación Científica y Bioestadística para profesionales y estudiantes deficiencias médicas. Santiago (Chile): RiL Editores.

Ekelund, R. \& Hebert, R. (2008). Historia de la teoría económica y de su método (4. ${ }^{\mathrm{a}}$ ed.). México: Editorial McGraw-Hill.

Fernández, A. Rodríguez, L. y Parejo J. (2008) Política Económica. México: Editorial McGraw-Hill.

Guerra, José. (2008). La inflación en Venezuela: propuestas para su enfrentamiento. Caracas: Publicaciones del Instituto Latinoamericano de Ciencias Sociales.

Guerra, José. (2013). El legado de Chávez. Un análisis de la economía venezolana y sus posibilidades. Venezuela: Editorial Libros X Marcados.

Herrerías, Armando. (2011). Historia del pensamiento económico. México: Editorial Limusa.

Manzano, O.; Méndez, R.; Pineda, J. \& Ríos, G. (2008). Macroeconomía y petróleo. México: Pearson Education.

Martínez M. (2013). Epistemología y Metodología Cualitativa en las Ciencias Sociales. México: Trillas. 


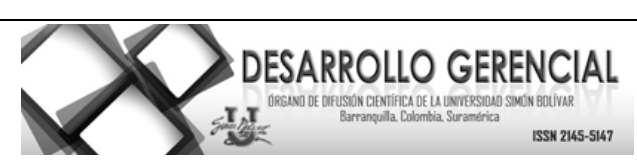

Mochón F. (2008). Introducción a la macroeconomía. México: Editorial McGraw-Hill.

Nieto, S. \& Rodríguez, M. (2009). Investigación y evaluación educativa en la sociedad del conocimiento. España: Ediciones Universidad de Salamanca.

Oficina Nacional de Presupuesto. (2014). Clasificación presupuestaria de recursos y egresos. Extraído el 5 $\begin{array}{llll}\text { de } & \text { agosto } & 2014 & \text { desde }\end{array}$ http://www.onapre.gob.ve/index.php/publicaciones/descargas/viewcategory/30-clasificadorespresupuestarios.

Toro, José. (1993). Fundamentos de teoría económica. Un análisis de la política económica venezolana. Caracas: Editorial Panapo.

Zabala, D. \& González, A. (1992). Tratado moderno de economía. Caracas: Editorial Panapo. 